\title{
HANS FRANK E L'ISTITUZIONALIZZAZIONE DI UN REGIME
}

\author{
EMILIANO VITTI (*)
}

Nota presentata dal m.e. Silvio Beretta

(Adunanza del 25 giugno 2015)

«ll potere e la certezza di poter usare

la violenza senza alcuna resistenza

sono il veleno più dolce e più pernicioso

che possa essere propinato a un governo.

Questo veleno diventa alla lunga mortale

e la storia insegna, infatti,

che $i$ sistemi giuridici durano secoli,

mentre $i$ sistemi basati sulla violenza

durano solo decenni».

Hans Frank

SunTO. - Con la fine della Campagna di Polonia che apriva lo scenario bellico della seconda guerra mondiale, i tedeschi assunsero il controllo della porzione di territorio assegnata loro dagli accordi germano-sovietici dell'agosto-settembre 1939, conferendogli una struttura subordinata al Reich sul piano istituzionale, giuridico, socio-economico nonché con funzioni e obiettivi specifici da raggiungere, come il riordino del sistema amministrativo del territorio attraverso l'istituzione del Governatorato generale di Polonia. Ogni riflessione storiografica non può comunque prescindere dall'analisi del ruolo del Governatore Hans Frank. Di primaria importanza furono infatti le caratteristiche della sua carica come conseguenza della formazione culturale e professionale di Frank, della sua devozione a Hitler, delle incertezze personali, del suo ruolo di giurista del Reich nonché di avvocato personale del Führer prima del conferimento della carica di Governatore. La necessità di una gestione del territorio congiunta con le SS provocò inoltre attriti e conflitti

(") Università degli Studi di Pavia, Italia.

E-mail: emiliano.vitti01@universitadipavia.it 
di competenza, sia a livello politico-istituzionale sia sul piano personale con alcuni degli ufficiali responsabili per conto di Heinrich Himmler. L'analisi di questa entità territoriale atipica appare fondamentale per comprenderne i meccanismi e per fornire un quadro accettabilmente completo dell'amministrazione di uno "Stato" sui generis.

$$
* * *
$$

ABSTRACT. - At the end of September 1939 Polish Campaign opened the Second World War and Germans took control of their portion of territory, according to the German-Soviet agrements of August-September 1939, giving it a juridical, institutional and socialeconomic structure subject to the Reich, with specific functions and aims, as the reorganization of the administrative system through the institution of the General Government of Poland. Nevertheless, every historiographical reflection cannot be separated from the survey of Governor Hans Frank's role. His position was in fact relevant owing to his cultural and professional education, his loyalty to Hitler, his personal uncertainties, his role of First Reich's Jurist and private Hitler's lawyer, before becoming Governor of Poland. The need of a management of Poland with SS caused frictions and jurisdiction conflicts, both at the political-institutional and on a personal level with some of the main responsible officials on behalf of Heinrich Himmler. The analisys of these atypical territorial entity through an approach which was technically correct before humanitarian is basic to understand inner workings and to try to produce a framework of the system as complete as possible about the administration of these sui generis "State".

Con la fine della Campagna di Polonia che apriva lo scenario bellico della seconda guerra mondiale, i tedeschi presero il controllo della loro porzione di territorio secondo gli accordi germano-sovietici del 23 agosto 1939 e le modifiche apportate in data 28 e 29 settembre e gli diedero un aspetto subordinato al Reich sul piano istituzionale, giuridico, socio-economico e con funzioni e obiettivi specifici da raggiungere. Il piano di lavoro degli occupanti prevedeva il riordino del sistema amministrativo del territorio attraverso l'istituzione del Governatorato generale di Polonia, la risoluzione di alcune controversie giuridiche sul piano interno e la gestione degli aspetti economici e umanitari che ne caratterizzarono la vita negli anni 1939-1942. Ogni riflessione storiografica non può prescindere dall'analisi del ruolo del Governatore Hans Frank. Di primaria importanza furono le caratteristiche della sua carica come conseguenza della propria formazione culturale e professionale, la sua devozione a Hitler, le incertezze personali, il ruolo di giurista del Reich e avvocato personale del Fübrer prima del conferimento della carica di Governatore.

Costituiscono inoltre elementi fondamentali l'organizzazione del governo sul territorio e il sistema di trattamento degli ebrei e dei polacchi "residenti"; la necessità di una gestione congiunta delle questioni razziali con le SS provocò attriti e conflitti di competenza sia a livello 
politico-istituzionale sia sul piano personale con alcuni degli ufficiali responsabili per conto di Heinrich Himmler.

La scelta di questi temi deriva da un mio interesse per la storia del nazionalsocialismo e dal desiderio di studiare le politiche del regime nell'ambito giuridico, del lavoro, della pianificazione economica e della dottrina razziale; credo sia fondamentale un approccio corretto sul piano tecnico prima che su quello umanitario, per comprendere i meccanismi e cercare di fornire un quadro di sistema il più completo possibile sulla gestione di uno "Stato" sui generis.

\section{Divisione della Polonia}

Lo scoppio della guerra aveva lasciato la Polonia «sommersa da una burrasca» ${ }^{1}$ a causa del «risveglio dell'imperialismo tedesco, [che] tende[va] a stabilire la sua egemonia su tutto il continente europeo. Per ottenere questa egemonia la Germania doveva creare un blocco potente nel centro dell'Europa». ${ }^{2}$ La resa della Polonia del 6 ottobre e la successiva riorganizzazione del suo territorio rappresentarono i primi passi in questa direzione. ${ }^{3}$ I pensieri annotati da Joseph Goebbels sul suo diario il 10 ottobre 1939 sono indicativi: «La Polonia è finita. Nessuno parla più della restaurazione del vecchio stato polacco». ${ }^{4}$ Infatti, oltre alla sconfitta militare, anche l'assetto istituzionale polacco attraversava una fase di instabilità giuridica dettata dalla fuga del governo in Romania, alla quale venne posto rimedio con le dimissioni del Presidente Mosicki $i^{5}$ e al trasferimento dei suoi poteri supremi a Władysław Raczkiewicz, residente in Francia. Raczkiewicz assunse la carica il 30 settembre 1939, in ottemperanza alla legge costituzionale del 23 aprile 1935 e secondo la designazio-

1 Messaggio di Mosicki alla nazione polacca, in Relazioni Internazionali, V, 939, vol. XVII, n. 38, 740-1.

2 S.i.a., Intervista alla «Gazette de Lousanne» di un'alta personalità polacca, in Relazioni Internazionali 42, 17 (1939), 865-6.

3 J.-M. Le Breton, Una storia infausta: l'Europa centrale e orientale da 1917 al 1990, Bologna, Il Mulino, 1997, 220-1.

4 I diari di Goebbels 1939-1941, a cura di F. Taylor, Milano, Sperling \& Kupfer, $1984,27$.

5 Ignacy Mosicki (1867-1946), presidente della repubblica polacca dal $1^{\circ}$ giugno 1926 al 30 settembre 1939. 
ne del presidente dimissionario in data 17 settembre $1939 .{ }^{6}$ Il nuovo capo dello stato congedava il governo in esilio e nominava primo ministro il generale Władysław Sikorski, con Augustow Zaleski al dicastero degli esteri, carica già ricoperta dal 1926 al 1932; le personalità principali del nuovo governo «erano tutte della vecchia guardia pilsudskiana». ${ }^{7}$ Venne garantita la continuità delle alleanze antecedenti alla crisi, assieme all'impegno di non concludere accordi di pace separata. ${ }^{8}$ Il primo atto del governo Sikorski fu, il 2 ottobre, quello di protestare contro la spartizione territoriale della Polonia, conseguente agli accordi russo tedeschi del 2829 settembre. A questo proposito, i rapporti con l'Unione Sovietica si mostrarono altrettanto difficili: il 24 ottobre vi fu una protesta formale contro l'annessione da parte sovietica della Galizia e una protesta generale presso il segretario della Società delle Nazioni il 31 ottobre. Era intendimento del nuovo governo polacco chiarire le posizioni con l'Unione Sovietica, con la quale non vi erano «formali rapporti di belligeranza». ' L'intervento dell'Armata rossa infatti accentuò la gravità di una situazione già compromessa per la Polonia e le sue istituzioni statuali. Sembrava che i russi, nei primi giorni di occupazione, rispettassero le apparenze nella loro zona di influenza poiché - forse - buona parte dei residenti nella Polonia orientale appartenevano alle comunità ucraina e bielorussa. ${ }^{10}$ Per i polacchi il destino fu differente: coloro che avevano posizioni sociali di rilievo o un livello alto di istruzione furono imprigionati, spesso torturati e poi spediti nei campi di concentramento e di lavoro in Siberia o nel nord-est della Russia europea. ${ }^{11}$

Coloro che rimasero nelle zone amministrate dai tedeschi furono sottomessi ad una forma di governo particolare, il Governatorato generale di Polonia ${ }^{12}$ (Generalgouvernement für die besetzten polnischen Gebiete), istituito per decreto personale da Hitler il 12 ottobre 1939 e operativo dal 26 ottobre dello stesso anno.

6 L.C., Attività del governo nominale polacco, in Relazioni Internazionali 44, 18 (1939), 916.

7 Ibid.

8 Ibid.

9 Ibid. 1990, 221.

10 J.-M. Le Breton, Una storia infausta: l'Europa centrale e orientale da 1917 al

11 Ibid., 223.

12 In seguito GG. 


\section{QUESTIONI RAZZIALI E SFRUTTAMENTO}

Secondo la legge sulla «unità del partito e dello Stato» del $1^{\circ}$ dicembre 1933, integrata dall'ordinanza del Führer del 29 marzo 1935, il partito era «il depositario dell'idea tedesca dello stato ed [era] indissolubilmente unito allo stato», ${ }^{13}$ diventando così un ente di diritto pubblico guidato dal dittatore. Le funzioni e l'autorità del partito erano circoscritte dalla legge e le sue attività erano controllate dallo Stato. ${ }^{14}$

Secondo Hans Frank la Germania non doveva rappresentare solo lo stato nazionalsocialista, ma essere l'esempio dell'attuazione del principio del Führerstaat, ossia di uno «Stato impersonato e diretto dal Führer, nelle direttive del partito nazionalsocialista, per i fini e secondo le volontà del popolo germanico». ${ }^{15} \mathrm{Il}$ decreto personale fu lo strumento legislativo più usato da Hitler. In questo modo il 7 ottobre 1939 nominò Himmler commissario per il rafforzamento della razza tedesca, con pieni poteri di perseguire la «germanizzazione» e la «purificazione razziale» dei territori orientali occupati. ${ }^{16}$ Il Reichsfübrer delle SS costruì un apparato con finalità di controllo nelle deportazioni di massa dei gruppi etnici non germanici. Questi “indesiderabili” erano destinati al GG (in polacco Generalna Gubernia - governo generale). Il territorio occupato fu usato come contenitore di "indesiderabili" e "subumani" dal quale il Reich attinse manodopera coatta per sostenere l'economia di guerra. ${ }^{17}$

Il Führer era «interprete e plenipotenziario generale del popolo germanico». ${ }^{18}$ All'interno della Volksgemeinschaft (comunità popolare) doveva «essere riconosciuto l'ordinamento primario, che [aveva] la funzione di conservare la stirpe e di renderla capace di opere civili». ${ }^{19}$ Le politiche razziali attuate nella Polonia occupata derivavano dalla dottrina nazionalsocialista e dalla produzione di normative minuziosa-

13 F. Neumann, Behemoth. Struttura e pratica del nazionalsocialismo, Milano, Bruno Mondadori, 1999, 77.

14 Ibid.

15 H. Frank, Fondamento giuridico dello Stato nazionalsocialista, Milano, Giuffrè, $1939,8$.

16 I. Kershaw, Hitler e l'enigma del consenso, Roma, Laterza, 2004, 181.

17 Ibid.

18 H. Frank, Fondamento giuridico dello Stato nazionalsocialista, cit., 8.

19 Ibid. 
mente studiate e atte a disciplinare i rapporti tra tedeschi, polacchi e alcune categorie giuridiche intermedie: regolamento sulle adozioni nelle liste popolari tedesche (Deutsche Volksliste ${ }^{20}$ ) del 28 ottobre 1939, subito dopo la creazione del GG; decreto di Himmler del 12 settembre 1940 sulla supervisione e selezione razziale della popolazione del GG; regolamento sulle DVL per i territori occupati del 4 marzo $1941 .^{21}$

Il processo venne chiamato nel suo insieme «Exklusion und Inklusion». ${ }^{22}$ I criteri, passati dalla dottrina alla formazione, sono stati gestiti dal legislatore tedesco in modo attento ma non senza lacune. Data la valenza pseudo-scientifica delle teorie nazionalsocialiste sulla razza, non era possibile abbracciare in un arco normativo tutta la casistica, rendendola insieme credibile e giustificativa degli obiettivi del regime. Per esempio la varietà di etnie che abitavano i territori orientali (die Verschiedenartigkeit der Bevölkerung in den eingegliederten Ostgebiete) rendeva in molti casi inattuabili le disposizioni di legge che dovevano includere ed escludere le "altre razze" da quella ariana. ${ }^{23}$

L'inadeguatezza dell'ideologia nazista nel sottostare a precise normative - pur redatte $a d h o c$ - e quindi a realizzare una parte importante della Weltanschauung hitleriana veniva colmata con la brutalità delle azioni nei territori orientali, «onde assicurare con la massima fermezza i diritti tedeschi» ${ }^{24}$ Le differenti istituzioni che se ne occupavano entravano spesso in conflitto tra loro; le questioni più frequenti si concentravano sui territori polacchi (Warthegau, Alta Slesia Orientale, Danzica e Gdynia, GG), nei quali si presentavano problemi di tipo etnico. Molti polacchi avevano i requisiti razziali per entrare a far parte della DVL; ne possedevano perciò i requisiti fisici, di sangue e territoriali, in base alle disposizioni del diritto razziale pangermanico. ${ }^{25}$

Ogni cittadino tedesco discendente da famiglia tedesco-ariana era

20 In seguito DVL.

21 B. Kundrus, Regime der Differenz. Volkstumspolitische Inklusionen und Exklusionen im Warthegau und im Generalgouvernement 1939-1944, in Volksgemeinschaft. Neue Forschungen zur Gesellschaft des Nationalsozialismus, a cura di Frank Bajohr e Michael Wildt, Frankfurt am Main, S. Fischer Verlag, 2009, 114.

22 Ibid., 107.

23 Ibid., 108.

24 I. Kershaw, Hitler e l'enigma del consenso, cit., 192.

25 B. Kundrus, Regime der Differenz. Volkstumspolitische Inklusionen und Exklusionen im Warthegau und im Generalgouvernement 1939-1944, cit., 114. 
un tedesco puro in qualunque parte del mondo; per coloro che non possedevano la purezza necessaria furono stabiliti quattro livelli di classificazione o «appartenenza» (Zugebörigkeit). Nel gruppo 1 erano compresi i soggetti attivi nella Volkskampf (lotta popolare), parenti di cittadini tedeschi, con tutte le carte in regola per appartenere alla Volksgemeinschaft tedesca. Nel gruppo 2, le persone che avevano mantenuto la loro germanicità acquisivano la cittadinanza tedesca, con effetto retroattivo a partire dalla data di annessione al Reich dei territori polacchi in cui vivevano. Il gruppo 3 comprendeva i cittadini di origine tedesca che si erano legati negli anni alla cultura polacca ma dei quali Himmler riconosceva tuttavia il pieno valore razziale; queste persone venivano inserite nel gruppo dei tedeschi da rieducare alla deutsche Volksgemeinschaft. Alla fine della rieducazione il RuSHA (SS-Rasse und Siedlungshauptamt), l'ufficio centrale per la razza e le colonie, di concerto con il RKFDV (Reichskommissariat für die Festigung deutschen Volkstums), il commissariato del Reich per il rafforzamento della germanicità, consegnava a questi cittadini idonei alla cultura tedesca i moduli per l'ottenimento della cittadinanza a termine (Staatsbürgerschaft auf Wiederuf). Secondo la legge, per dieci anni la cittadinanza era a tempo e poteva essere anche ritirata se il livello di germanicità riconosciuto al soggetto non veniva mantenuto costante. Nel gruppo 4 venivano inseriti coloro che, pur non essendo tedeschi puri ma cittadini polacchi, avevano nell'albero genealogico origini tedesche tali da essere compresi in un processo di naturalizzazione (Einbürgerungsverfahren) al termine del quale i soggetti potevano ricevere la cittadinanza a termine. ${ }^{26}$

Coloro che rientravano nei gruppi 3 e 4 potevano essere definiti «appartenenti al popolo in formazione» (Volksgenossen im Lernprozess). ${ }^{27}$

Il processo era soggetto a valutazione individuale e non collettiva. I funzionari del RKFDV dovevano valutare i "cittadini potenziali" in considerazione delle origini tedesche e non del passato da cittadini polacchi. In caso di valutazione positiva i soggetti usufruivano di un sistema di protezione particolare (Schutzangebörige), che poneva questi cittadini polacchi in una condizione giuridica di livello superiore (rech-

26 Ibid., 114-5.
27 Ibid. 
tlicher Zustand). Secondo i parametri legislativi creati ex-novo dal legislatore del Reich andava attuato un «urgente e necessario progetto di germanizzazione», ${ }^{28}$ il cui obiettivo era integrare i soggetti meritevoli e introdurli per scaglioni nel sistema di istituzioni e servizi fondamentali del Reich sul piano sociale, politico e culturale. I cittadini avevano diritto all'assistenza sanitaria e alimentare e all'istruzione pubblica.

Nel quinquennale periodo di controllo dei valori di cittadinanza, i beni degli appartenenti al gruppo 4 come case, fattorie, fabbriche e la loro documentazione passavano in amministrazione fiduciaria allo stato; per i polacchi e gli ebrei c'era invece il provvedimento definitivo della confisca di tutti i beni. Le «regole necessarie» (Regelungsbedarf) servivano alla struttura burocratica delle SS per gestire il passaggio attraverso la disciplina di ogni singolo aspetto della vita delle «persone non completamente pure» soggette a giudizio. ${ }^{29}$

Uno degli scopi dello stato tedesco in questi processi di classificazione era l'acquisizione di forze giovani per il servizio di leva; agli uomini ritenuti idonei veniva quindi imposto l'obbligo del servizio militare. Il $3 \%$ degli appartenenti al gruppo 3 entrava a far parte della Webrmacht. ${ }^{30}$

Inoltre, istituzionalizzando le visioni ideologiche sulla razza era possibile per lo stato acconsentire alle unioni miste (Mischehen) che, se monitorate e certificate dall'ufficio competente, avrebbero concorso a formare e ampliare la Großdeutsche Volksgemeinschaft. ${ }^{31}$

Questa distinzione tra diritti e scopi di individui "recuperabili" e la totale assenza di considerazione verso gli Untermenschen slavi e gli ebrei aveva l'importanza che si da a un'accorta politica aziendale sulla conservazione di un alto livello qualitativo di popolamento dei territori occupati (bevölkerungspolitischen Mikromanagement). ${ }^{32}$

Vi erano importanti passaggi di uomini tra Warthegau e GG in base alle delibere delle commissioni operanti sulla classificazione razziale, per una accurata selezione e per relegare tutti gli «indesiderabili» nel GG. Il compito del Governatore fu sotto questo aspetto l'amministrazione di una «pattumiera razziale e sociale del Reich».33

\begin{tabular}{ll}
\hline 28 & Ibid. \\
29 & Ibid., 115. \\
30 & Ibid., 116. \\
31 & Ibid., 117. \\
32 & Ibid., 116. \\
33 & Ibid.
\end{tabular}


Diceva Frank nel 1940: «ai polacchi devono essere messe a disposizione solo quelle possibilità di istruzione che possono mostrare loro la mancanza di prospettive del destino del loro popolo [...] dovranno capire la differenza tra il tenore di vita del popolo dominante e quello dei popoli soggetti $[\ldots]$ nessun polacco dovrà superare il rango di un capo operaio».34

A partire dal 21 marzo 1942 il ruolo del GG di territorio di raccolta della forza lavoro coatta venne ricoperto da Frank come collaboratore del ministro della produzione bellica Albert Speer, organizzatore e ottimizzatore dei processi industriali e del reclutamento della manodopera, nonché di Fritz Sauckel,,$^{35}$ responsabile plenipotenziario per la distribuzione del lavoro (Generalbevollmächtiger für den Arbeitseinsatz). Anche il riconoscimento delle coppie miste e il loro trasferimento forzato dal GG in Germania si inquadravano nel processo di incremento della forza lavoro utile al sostegno dell'economia di guerra.

I numerosi ordini di divieto per i matrimoni misti presentavano una importante eccezione normativa. Su ordine di Frank, la registrazione avveniva solo dopo l'approvazione e la compilazione dei relativi moduli di autorizzazione; i casi diversi erano pochi, derivavano solo dall'alto livello di appartenenza di sangue del marito e si svolgevano in concomitanza con il trasferimento forzato nei territori del Reich. Il marito tedesco di una moglie polacca o ucraina doveva presentare alle autorità del partito o delle SS presso la sede del GG un apposito certificato di idoneità matrimoniale assieme a un modulo di adesione da richiedere agli uffici del GG. L'abbandono del GG subito dopo aver ottenuto i documenti matrimoniali era una regola da seguire scrupolosamente. Le pene per i trasgressori o i ritardatari potevano arrivare, nei confronti dei dipendenti pubblici, al licenziamento con conseguente perdita dei diritti pensionistici. ${ }^{36}$

Le eccezioni che creavano livelli intermedi (Zwischenschichten) nelle normative sulla disciplina della razza venivano applicate solo nel GG, consentendo così alle coppie di rientrare nel Warthegau "in regola”, poiché la politica di unità popolare non veniva alterata se l'eccezione si verificava sotto l'amministrazione di Frank anziché in una unità ammi-

34 K.D. Bracher, La dittatura tedesca, Bologna, Il Mulino, 1973, 550.

35 Nominato il 21 marzo 1942.

36 B. Kundrus, Regime der Differenz. Volkstumspolitische Inklusionen und Exklusionen im Warthegau und im Generalgouvernement 1939-1944, cit., 117. 
nistrativa del Reich. Si consentiva così, per questioni pratiche (le famiglie numerose potevano fornire più forza lavoro e più soldati potenziali per il fronte, necessari specialmente dopo l'inizio della campagna di Russia), un alto grado di flessibilità sulla regolamentazione del matrimonio. ${ }^{37}$ Erano molti i gerarchi delle SS che spingevano per fare ammettere un numero alto di persone nelle quattro categorie classificate, con alcune opinioni contrarie sul piano ideologico. L'Obergruppenfübrer Richard Hildebrandt, comandante del RuSHA dal 20 aprile al 25 dicembre 1943 e intransigente sostenitore dell'unicità dell'appartenenza pura al sangue ariano, sosteneva: «...einmal Pole, immer Pole»..$^{38}$ Nonostante queste posizioni, l'attuazione dei decreti sulla razza e il matrimonio ebbero una notevole efficacia nel dare flessibilità alle politiche razziali e furono fondamentali per realizzare la Volkstumspolitik (traducibile come "politica della nazionalità").

Nel nuovo ordinamento giuridico nazionalsocialista il popolo aveva un ruolo fondamentale: la sua natura giuridica possedeva un'aura divina. ${ }^{39}$

Nella rivisitazione dello Ius divinum ecclesiastico, Frank affermava tre «verità giuridiche» essenziali nell'ottica nazionalsocialista:

...1) la storia della creazione ci insegna che l'umanità era divisa in gruppi o razze originarie, concetto col quale vogliono essere indicati quei primordiali aggruppamenti della storia della umanità, fra loro differenziabili per il modo di vivere e per il fondamentale orientamento spirituale, derivanti immediatamente ed esclusivamente da una evoluzione naturale (criterio del sangue) ...; 2) i popoli sono la fusione degli elementi razziali originari in una vita comune, storicamente determinata, secondo caratteristiche e secondo leggi proprie. Un popolo può essere composto di più razze originarie ...;3) i punti di partenza razziali di un popolo devono essere però della stessa specie, ossia soltanto le combinazioni di razze non contrastanti fra loro possono svilupparsi storicamente fino al grado di popoli capaci di grandi opere. ${ }^{40}$

Anche Himmler era convinto che esistessero degli individui non appartenenti alla razza ariana o addirittura completamente slavi, polacchi

\footnotetext{
37 Ibid., 117-8.

38 Ibid., 122.

39 H. Frank, Fondamento giuridico dello Stato nazionalsocialista, 24.

40 Ibid., 24-5.
} 
e ucraini specialmente, aventi caratteristiche tali da renderli "arianizzabili”. L'arianizzazione (Arisierung) di soggetti slavi comprendeva casi di bambini polacchi e ucraini sottratti alle proprie famiglie e dati in adozione a coppie ariane sterili. ${ }^{41} \mathrm{Nel}$ discorso del 4 ottobre 1943 a Posen, Himmler dichiarò:

...Ciò che le nazioni ci possono dare, per quanto riguarda un sangue dello stesso tipo del nostro, noi lo prenderemo, se necessario, portando via i loro bambini e educandoli qui, insieme ai nostri. Che le nazioni vivano in prosperità o muoiano di fame come bestie, a me importa solo nella misura in cui avremo bisogno degli appartenenti ad esse come schiavi della nostra Kultur, altrimenti per me sono prive di ogni interesse. $^{42}$

La questione razziale riguardante invece la grande maggioranza dei "normali" polacchi doveva essere risolta al di fuori di qualunque vincolo di legalità, sul piano patrimoniale come su quello umanitario. Le espropriazioni, le confische, le deportazioni a scopo di sfruttamento del lavoro, la vita nei ghetti, lo sterminio furono gli aspetti in cui la brutalità pretesa da Hitler si applicò alla popolazione slava ed ebraica. Secondo le dichiarazioni dello stesso Hitler fatte a Keitel ${ }^{43}$ nel 1942 «tali metodi sarebbero [stati] incompatibili con i principi cui in altre occasioni aderi[vano] [i tedeschi] $\gg^{44}$

Le obiezioni di alcuni ufficiali contro i metodi adoperati furono liquidati da Hitler come «infantili lamentele» di capi militari che intendevano affrontare la guerra con «metodi da esercito della salvezza». ${ }^{45}$ Le terre polacche occupate annesse al Reich nel 1940, intenzio-

41 Riferimenti al processo di arianizzazione si trovano in I. Kershaw, Hitler e l'enigma del consenso, 169-203.

42 Nazi Conspirancy and Aggression (in seguito NCA) 4, 559 (Nuremberg Documents, in seguito ND, PS-1919).

43 Feldmaresciallo Wilhelm Keitel (Helmscherode, 22 settembre 1882 Norimberga, 16 ottobre 1946), comandante dell'OKW (Oberkommando der Webrmacht) dalla sua istituzione, il 4 febbraio 1938, alla fine della guerra.

44 Der Prozess gegen die Hauptkriegsverbrecher vor dem Internationalen Militärgerichtshof: angewandten Linguistik und Translatologie, 1862-7056, Band 9, Nürnberg, 1947-1949, 379.

45 Heeresadjutant bei Hitler 1938-1943. Aufzeichnungen des Majors Engel, a cura di H. von Kotze, Stuttgart, Deutsche Verlags-Anstalt, 1982, 68. 
nalmente escluse dai vincoli del diritto penale tedesco, divennero aree di sperimentazione del Nuovo Ordine (Neue Ordnung). Parallelamente al compito di Frank di comandare senza poteri uno stato debole che fosse il luogo di raccolta delle "razze inferiori", Albert Forster e Arthur Greiser, ${ }^{46}$ i due Gauleitern di Danzica-Prussia Occidentale e Wartheland, assunsero un ruolo significativo nel quadro politico e amministrativo del Großdeutsches Reich. Il compito affidato da Hitler ai suoi amministratori locali nel 1939 fu la germanizzazione completa dei territori polacchi in 10 anni, con libertà d'azione sugli strumenti da adoperare. ${ }^{47}$ I reparti operativi speciali (Einsatzgruppen) del servizio di sicurezza di Reinhard Heydrich lavorarono all'annientamento dell'inteligencja polacca ${ }^{48}$ come parte della «azione straordinaria di pacificazione» (Außerordentliche Befriedungsaktion o AB-Aktion) finalizzata a stabilizzare la politica tedesca di occupazione (Besetzungspolitik). ${ }^{49}$ Greiser finanziava il piano di germanizzazione attraverso gli espropri e le confische di beni immobili e il congelamento dei conti correnti bancari degli ebrei e dei polacchi. Poco prima della sua investitura ufficiale, il 5 ottobre 1939, ordinò al commissario cittadino Gerhard Scheffler la confisca di «edifici, terreni e appartamenti nell'area municipale di Posen» ${ }^{50}$ da destinare ad uso amministrativo e per l'interesse economico della Germania. $^{51}$

Inoltre l'opera di "rieducazione" nazista dei polacchi venne propagandata come una giusta reazione della Germania all'ottusità dei polacchi, i quali non vollero, secondo Goebbels, «udire la generosa

46 Albert Forster (Fürth, 26 luglio 1902 - Varsavia, 28 febbraio 1952), Gauleiter di Danzica-Prussia Occidentale dal 2 novembre 1939 al marzo 1945; Arthur Greiser (Schroda, Posen, 22 gennaio 1897 - Poznan, 21 luglio 1946), Gauleiter dall'8 ottobre 1939 e Reichsstattalter (governatore locale) del Wartheland dal 29 gennaio 1940 fino alla fine della guerra.

47 I. Kershaw, Hitler e l'enigma del consenso, 192.

48 H. Frank, Deutsches Verwaltungsrecht, Münich, Franz Eher Nachfolger, 1937, 420-1-4-5-30.

49 I. Kershaw, Hitler e l'enigma del consenso, 195.

50 C. Epstein, Model Nazi. Arthur Greiser and the occupation of Western Poland, New York, Oxford University Press, 2010, 232.

51 Come per il GG, anche nel Wartheland una buona parte del bilancio del Gau proveniva dagli espropri e dallo sfruttamento della manodopera coatta dei polacchi. Cfr. ibid., 231. 
offerta del Fübrer $\rangle^{52}$ e non analizzarono attentamente le "vantaggiose" proposte tedesche perché chiusi nel pregiudizio anti-germanico. ${ }^{53}$

Per questo i territori appartenuti al disciolto stato polacco avevano bisogno di un "nuovo regime", di un'opera di adattamento e di «sana emulazione ${ }^{54}$ da parte dei nuovi distretti amministrativi per raggiungere l'alto grado di sviluppo e produzione previsto dai piani nazionalsocialisti. Lo spirito nuovo nella gestione dei territori polacchi derivava dalla concezione spaziale della dottrina del Reich e interessava il redditizio sfruttamento sia dei territori acquisiti direttamente dalla Germania attraverso l'annessione, sia di quelli controllati indirettamente (GG) ${ }^{55}$ Secondo la propaganda del Völkischer Beobachter, ${ }^{56}$ il GG era la «sede nazionale dei polacchi» e rientrava nella «sfera d'influenza della potenza e dell'ordine germanico». ${ }^{57}$ Il 26 ottobre 1939 divenne «il giorno iniziale della riorganizzazione in Oriente». ${ }^{58} \mathrm{E}$ molto importante sottolineare che il VB definiva il GG una «organizzazione statale speciale, formalmente autonoma ma di fatto posta sotto la sovranità tedesca»:59 questa descrizione contrasta in parte con i proclami del giurista Frank che, per tutta la durata del suo mandato, rivendicò l'autonomia formale e giuridica dal Reich per la carica di Generalgouverneur, definendola come dipendente solo dal Führer e in grado di produrre ed emanare leggi o atti aventi forza di legge come un capo di stato qualsiasi. ${ }^{60}$

La propaganda nazista presentava all'opinione pubblica il GG come «il primo esempio del diritto di comando che spetta alla Germania di fronte ai gruppi etnici stranieri», per «soccorrere quelle classi polacche che in passato dovettero soffrire per la incapacità del proprio governo e per la mancanza di principi organizzativi». ${ }^{61} \mathrm{Il} \mathrm{VB}$,

52 Discorso del ministro Goebbels a Kattowitz, in Relazioni Internazionali 35, 18 (1940), 1367.

53 Ibid.

54 S.i.a., Un nuovo regime nelle regioni ex-polacche, in Relazioni Internazionali 43, 18 (1939), 1584.

55 Ibid.

56 In seguito VB.

57 S.i.a., Un nuovo regime nelle regioni ex-polacche, cit., 1584.

58 Ibid.

59 Ibid.

60 Cfr. C. Klessmann, Der Generalgouverneur Hans Frank, in Vierteljährhefte für Zeitgeschichte, 3 (1971), 245-60.

61 S.i.a., Un nuovo regime nelle regioni ex-polacche, 1585. 
nel quadro della "normalizzazione" del sistema polacco prevista dal programma nazionalsocialista, parlava di «completa eliminazione della disoccupazione polacca [...] riattivazione delle industrie [...] riordinamento dei rapporti sociali mediante misure preventive»:.2 nella realtà che non veniva resa nota all'opinione pubblica tedesca la disoccupazione veniva "sconfitta" mediante la schiavizzazione; la ripresa del sistema industriale venne attuata in funzione dell'asservimento di tutto l'apparato produttivo polacco alle esigenze tedesche; le misure preventive in campo sociale furono la ghettizzazione e la repressione del malcontento. Il 2 ottobre 1940 il Führer aveva espresso la sua opinione sulla sorte dei polacchi in un memorandum a Frank in cui scrisse:

I polacchi sono soprattutto adatti ai lavori umili ... Per loro un miglioramento è inconcepibile. In Polonia bisogna tener basso il tenore di vita, non si deve permettere che esso si innalzi ... I polacchi sono pigri e per farli lavorare bisogna usare mezzi coercitivi ... Il Governatorato generale deve servirci solo come una riserva di operai non qualificati ... Di là, ogni anno, potremo procurarci gli operai di cui il Reich abbisogna ... È indispensabile tener presente che l'alta borghesia e la piccola nobiltà polacche debbono cessare di esistere; ciò potrà sembrare crudele, ma esse vanno sterminate ovunque risiedano ... I polacchi devono avere un unico signore, il tedesco ... È la legge della vita. ${ }^{63}$

La propaganda tedesca sottolineava anche l'utilizzo fatto della bassa manovalanza polacca per realizzare l'ammodernamento e l'estensione della rete stradale, volta all'aumento del traffico merci e di mezzi dell'esercito. ${ }^{64}$

La propaganda esaltò l'alto livello qualitativo dell'assistenza sanitaria attraverso le vaccinazioni e la lotta alle malattie infettive: in realtà le epidemie divennero un problema grave dei ghetti, che specialmente dopo il 1941 ne decimarono la popolazione, senza che le autorità del GG impiegassero risorse economiche superiori a quelle necessarie a preservare l'integrità della manodopera polacca. Sul piano ideologico furono attuate «le radicali misure contro la piaga nazionale, costituita

\footnotetext{
62 Ibid.

63 NCA, vol. III, 798-9 (ND, PS-1130).

64 S.i.a., Un nuovo regime nelle regioni ex-polacche, 1585.
} 
dagli ebrei», ${ }^{65}$ intensificatasi dopo la conferenza di Wannsee del 20 gennaio 1942.

L'odio anti-polacco si manifestò sul piano umanitario e sul piano culturale: la Polonia venne depredata dei suoi tesori artistici, per ordine di Göring e su azione di Frank: «Mi sforzerò di spremere da questa nazione tutto ciò che è ancora possibile». ${ }^{66}$

Il GG avrebbe dovuto rappresentare un nuovo sistema di governo per prendere i provvedimenti necessari a risolvere le questioni elencate. Un decreto del Fübrer del 3 ottobre 1939 stabilì che la Polonia fosse amministrata «mediante uno sfruttamento senza scrupoli». Frank, nella sua qualifica di primo giurista del Reich, aveva il compito di costruire un nuovo sistema amministrativo che il VB definiva della «massima semplicità e della massima efficienza». ${ }^{67}$

L'esercizio dell'attività di governo nel GG, la struttura istituzionale, la gerarchia delle fonti del diritto e i rapporti con le altre autorità operanti sul territorio ebbero nei fatti caratteristiche diverse da quelle teorizzate dallo stesso Frank.

\section{Il GOVERnATORE E L’ASPETTO GIURIDICO}

Hans Frank nacque a Monaco nel 1900. Nel 1919 iniziò a studiare legge all'università di Monaco e di Kiel e contemporaneamente a dedicarsi alla politica. Nel 1922 entrò a far parte dei Freikorps Epp. ${ }^{68} \mathrm{Nel}$ 1923 partecipò al tentato colpo di stato di Hitler a Monaco. Dopo il fallimento dell'impresa si rifugiò per un breve periodo in Austria, ritornando in Germania nel 1924 per laurearsi in legge; da quel momento divenne il legale personale di Hitler. Si iscrisse formalmente al partito nel 1928 divenendone il giurista di riferimento. L'utilità di Frank decrebbe rapidamente con la conquista nazionalsocialista del potere: il suo ruolo di avvocato non era più di strategica importanza per il Fübrer.

65 Ibid.

66 Trials of Major War Criminals before the International Military Tribunal (in seguito IMT), vol. VIII, New York, AMS Press, 1947, 9.

67 S.i.a., Un nuovo regime nelle regioni ex-polacche, 1585.

68 Formazione paramilitare composta da veterani di guerra creata dal generale Franz von Epp (Monaco, 16 ottobre 1868-31 dicembre 1946). Caratterizzò la sua attività per la violenza delle azioni dimostrative e per il forte anti-marxismo. 
Ricoprì così cariche quasi onorifiche: nel 1933 ministro della giustizia del Land di Baviera, nel 1934 ministro senza portafoglio. Tra il 1934 e il $1941 \mathrm{fu}$ presidente della Accademia Tedesca di Giurisprudenza. ${ }^{69}$

Il 12 ottobre 1939 fu emanato da Hitler il decreto istitutivo del Generalgouvernement für die besetzen polnischen Gebiete (Governatorato generale per le aree occupate di Polonia), poi ridotto a Generalgouvernement Polens o semplicemente a Generalgouvernement. L'entrata in vigore delle disposizioni contenute nel decreto avvenne il 26 ottobre 1939; da questa data Frank fu insignito della carica, unica nel Reich e nei territori occupati, di Governatore generale..$^{70}$ Frank si insediò nel castello di Cracovia, comportandosi come «un monarca feudale imposto da Hitler alla Polonia». ${ }^{71}$

Come membro del partito Frank doveva

...innanzitutto [assolvere il compito di] orientare gli sforzi di tutta la sua organizzazione verso la creazione di un nucleo stabile ... della dottrina nazionalsocialista; secondariamente, di educare ... il popolo [polacco] a questa idea; in terzo luogo, di fare accettare al popolo, così educato, l'autorità dello stato. ${ }^{72}$

\section{Il 31 ottobre 1939 emise un decreto}

...al fine di combattere gli atti di violenza contro il Governatorato generale: art.1, chi commette un atto di violenza contro il Reich tedesco o contro il potere supremo tedesco, esercitato nel Governatorato generale, sarà condannato a morte; art. 2, chi deliberatamente arreca danni alle attrezzature delle autorità tedesche, ai beni che servono alle autorità tedesche per lavorare, oppure ai beni di servizio pubblico, sarà condannato a morte; art. 3 , chi incita o invoglia alla disobbedienza ai decreti o ai provvedimenti delle autorità tedesche, sarà condannato a morte; art. 4, chi commette un atto di violenza contro un tedesco per via della sua appartenenza alla nazione tedesca, sarà condannato a morte ... art. 6, l'istigatore e il complice saranno puniti come l'autore, l'atto tentato sarà punito come l'atto compiuto ... art. 9, chi, ricevendo un'informazione sull'intenzione di commettere un reato di cui ai

69 Cfr. C. Klessmann, Der Generalgouverneur Hans Frank, 245-9.

70 Ibid., 249-54.

71 M. Broszat, Nationalsozialistische Polenpolitik 1939-1945, Stuttgart, Deutsche Verlags-Anstalt, 1961, 71.

72 F. Neumann, Bebemoth. Struttura e pratica del nazionalsocialismo, 76. 
commi 1-5 rinuncerà a denunciarlo alle autorità o alla persona minacciata da quel reato, sarà condannato a morte. ${ }^{73}$

Frank, di fatto un vassallo di Hitler, trasformò il GG nel «Gau dei Vandali $\gg^{74}$ restando allo stesso tempo sempre fedele al proprio credo di uomo di legge. Hitler considerava però «tutti i giuristi [come] minorati mentali dalla nascita o destinati a diventarlo con il passare del tempo»; $;^{75}$ li definì inoltre «traditori del popolo» e dichiarò in un discorso al Reichstag del 26 aprile 1942 che «non si sarebbe dato pace finché ogni tedesco non si fosse convinto che essere giuristi era una vergognosa ignominia». ${ }^{76} \mathrm{I}$ continui rimandi di Frank al valore del diritto gli procurarono la definizione da parte del Fübrer di «un giurista come tutti gli altri». ${ }^{77} \mathrm{Da}$ un lato Frank sosteneva che senza l'appoggio del diritto «uno stato avrebbe perduto il contenuto morale per sprofondare negli abissi della notte e dell'orrore»; ${ }^{78}$ dall'altro constatava con orgoglio gli effetti di una serie di decreti grazie ai quali «sarebbero stati uccisi circa due milioni di ebrei». ${ }^{79}$ Non vi era nessuna prospettiva per i piani di riforma del diritto auspicati da Frank, il cui carattere debole e arrendevole a Hitler lo rese sempre più incline a professare la propria fede nella forza della nuova dottrina tedesca mentre dava ordini ed emanava decreti brutali e in palese contraddizione con il Frank "giurista" ${ }^{80}$

73 R. Szuchta, Campi tedeschi dei nazisti sulla terra polacca occupata durante la seconda guerra mondiale, Varsavia, Ed. Ministero degli Affari Esteri, in s.i.d., in http:// www.poloniaeuropae.eu/wp-content/uploads/2012/11/pagine-della-storia-polacca.pdf e in http://www.lecorticalabresi.it/index.php/lecorticalabresi/article/viewArticle9 (0405-2015), 11.

74 Conversazioni di Hitler a tavola 1941-1942. Raccolte al suo quartier generale da Henry Picker, a cura di G. Ritter, Milano, Longanesi e C., 1952, 225.

75 Ibid.

76 H. Frank, Im Angesicht des Galgens. Deutung Hitlers und seiner Zeit auf Grund eigener Erlebnisse und Erkenntnisse, München, Grafelfing F. A. Beck, 1953, 116. 77 Ibid., 146.

78 Riunione del NSRB (Nationalsozialistischer Rechtswahrerbund), Berlino, 19 novembre 1941, in IMT, 29, 479.

79 Riunione governativa a Cracovia, 24 agosto 1942, cfr. IMT, 29, 580.

80 Molti autori trattarono la debolezza caratteriale di Frank. Tra questi, ho esaminato Joachim Fest (Il volto del Terzo Reich), Eugene Davidson (Gli imputati di Norimberga. Hermann Wilhelm Göring, Rudolph Hess, Martin Bormann, Albert Speer... La vera storia di ciascuno dei ventidue fedelissimi di Hitler processati per crimini contro l'umanità dal Tribunale alleato), Christph Klessmann (Der Generalgouverneur 
Il Governatore era cosciente del suo compito di "disinfestare" il GG dai pidocchi e dagli ebrei e che questo fosse l'obiettivo primario del GG al pari del reclutamento di lavoro coatto e dello sfruttamento economico; per esempio il 30 maggio 1940, mentre era in atto la «azione di pacificazione» tesa a impedire con il terrore la resistenza polacca contro gli occupanti tedeschi, Frank impose che «qualsiasi tentativo da parte delle autorità legali di interferire con l'azione intrapresa con l'aiuto della polizia [sarebbe stato] considerato un tradimento verso lo stato e gli interessi tedeschi». ${ }^{81}$

Nel sistema nazionalsocialista lo Stato non era un organismo dotato di personalità giuridica, ma un ordinamento giuridico che aderiva alla volontà e alle esigenze del popolo e che trovava in Adolf Hitler «la sua necessaria organizzazione unitaria». ${ }^{82}$ Non era facile adattare queste linee guida ad uno stato che, almeno formalmente, non era la Germania. Una delle problematiche tecnico- giuridiche del GG riguardava gli obiettivi dell'ordinamento interno allo stato: il benessere della popolazione non era uno scopo da perseguire, quindi i conflitti di competenze tra il Governatore, le SS e le autorità politiche locali assunsero il carattere di una lotta perenne per il potere, sovrapponendo le normative e rendendo confusa e disorganizzata l'amministrazione dello stato. Frank ripeteva di essere titolato a prendere le decisioni finali per quanto si riferiva al GG, perché il suo potere era diretta emanazione della volontà di Hitler; quindi disobbedire a lui significava disobbedire al Führer. ${ }^{83}$ Esistevano solo due tipi di autorità nel GG: quella limitata della Webrmacht e quella assoluta del Governatore. ${ }^{84}$

Frank esercitò il comando sul territorio e sulla produzione normativa, avvalendosi di metodi barbari e contrari al diritto germanico, del quale in patria si riteneva orgogliosamente la guida. Il Governatore tentò di sostenere sia la forza imprescindibile del diritto sia la legittima-

Hans Frank), Ian Kershaw (Hitler e l'enigma del consenso), Dieter Schenk (Hans Frank: Hitlers Kronjurists und Generalgouvernement).

81 IMT, 12, 37.

82 H. Frank, Fondamento giuridico dello Stato nazionalsocialista, 9.

83 Cfr. E. Davidson, Gli imputati di Norimberga. Hermann Wilhelm Göring, Rudolph Hess, Martin Bormann, Albert Speer... La vera storia di ciascuno dei ventidue fedelissimi di Hitler processati per crimini contro l'umanità dal Tribunale alleato, Roma, Newton \& Compton Editori, 2003, 452.

84 Ibid., 452-3. 
zione dell'arbitrarietà dell'autorità dichiarando: «Il legislatore si ispira ai bisogni dell'epoca, di cui egli deve realizzare le finalità [...] gli uomini non fanno soltanto la storia, essi sol fanno anche le leggi». ${ }^{85}$

Egli amava ribadire che «le leggi passano, il diritto è eterno». ${ }^{86}$ Nel caso specifico del GG l'attività di governo basata sul "diritto" anziché su leggi chiare e pragmatiche diede luogo a fenomeni illeciti ma "tollerati" dall'autorità tedesca, come la corruzione di pubblici funzionari - primo fra tutti lo stesso Governatore - e il mercato nero. ${ }^{87}$

Il concetto di competenza giuridica e legislativa si contrappose nella pratica ai conflitti di attribuzione che si verificavano spesso nel GG. Si può affermare che dopo i primi dieci mesi di governo (novembre 1939 - settembre 1940) vi furono scontri politici tra organi dello stato. Frank uscì da queste dispute danneggiato nel prestigio e nell'autorità della sua carica: vide infatti diminuire progressivamente la sua capacità di comando e la sua credibilità anche in Germania. ${ }^{88}$

Le disposizioni del Governatore riguardavano in linea teorica tutti gli ambiti dell'amministrazione, ordinaria e straordinaria. Di fatto, gli elementi che condizionavano l'attività e l'autorità di Frank erano tre: oltre al già citato disprezzo di Hitler verso i giuristi, si possono riconoscere gli attriti con le SS e con il partito riguardo ai ghetti, al bilancio interno, al lavoro coatto e al piano quadriennale dell'economia tedesca (del quale Göring era il plenipotenziario) che venne esteso al GG.

Riguardo le lotte di potere l'attuazione dei principi giuridici nazionalsocialisti nel GG coincise con l'emersione delle incongruenze tra il concetto di amministrazione, quello di arbitrarietà e l'idea di unire l'ordinamento giuridico al destino di un popolo.

Per dimostrare al Führer di essere un ottimo esecutore della politica nazionalsocialista, senza esitazioni né pietà nei confronti dei "nemici del Reich", Frank agì spesso in modo talmente duro e brutale da ricevere le lamentele di alcuni capi-distretto o governatori locali:

...la feroce e spietata caccia all'uomo praticata dappertutto, nelle città e nelle campagne, nelle vie, nelle piazze, nelle stazioni, ha molto scosso il senso di sicurezza delle popolazioni. Tutti si sentono esposti al peri-

85 H. Frank, Fondamento giuridico dello Stato nazionalsocialista, 20.

86 Ibid.

87 Riguardo al mercato nero, vedi punto e.

88 H. Frank, Fondamento giuridico dello Stato nazionalsocialista, 20. 
colo di venir catturati all'improvviso e inaspettatamente, in qualsiasi luogo e in qualsiasi momento dalla polizia, e di essere mandati in un campo di raccolta. Nessuno dei parenti viene a sapere che cosa accade all'arrestato. (Cracovia, febbraio 1943) ${ }^{89}$

Il rapporto più teso del Governatore fu con lo SSObergruppenfübrer Friedrich Wilhelm Krüger, Höherer SS und Polizeifübrer (comandante supremo delle SS e della polizia) del GG.$^{90} \mathrm{Il}$ sistema vigente delle mezze competenze che si riunificavano solo al vertice del sistema concentrandosi nella figura del Fübrer divenne la causa di una completa disorganizzazione - che rientrava nei piani di Hitler - e diede adito a numerosi conflitti nei quali Frank spesso uscì sconfitto. ${ }^{91}$

Krüger, con la protezione di Himmler, perseguiva una propria politica, alla quale Frank tentò di opporsi con atteggiamenti più moderati e una linea improntata su criteri di collaborazione. Quando questo approccio morbido non funzionava, il Governatore passava però ai mezzi brutali che lo resero famoso. In base ai decreti emanati durante la sua carica, la nascita e lo sviluppo di un «diritto comunitario tedesco» ${ }^{92}$ non vennero mai raggiunti nel GG, dove si riconobbe l'attuazione di una vaga formula di diritto internazionale «di un paese sotto la sfera di influenza del Reich» che lasciava aperta ogni possibilità, garantendo al Governatore generale una certa sovranità. L'incarico affidatogli inizialmente da Hitler era di assumere

...l'amministrazione dei paesi occupati dell'est [all'ottobre 1939] con l'ordine speciale di sfruttare senza scrupoli tutto quel territorio come conquista di guerra e terra di saccheggio, smantellandone la struttura economica, sociale, culturale e politica. ${ }^{93}$

La linea distruttiva di questo incarico contrastava, però, con il meccanismo insito in ogni attività amministrativa, mirata a stabilire precisi ordinamenti; Frank si rendeva conto di quali fossero i danni potenziali

89 NCA, 6, 80 (ND, 1526-PS).

90 J. Fest, Il volto del Terzo Reich, Milano, Gruppo Ugo Mursia Editore, $1970,339$.

91 Ibid.

92 H. Frank, Im Angesicht des Galgens. Deutung Hitlers und seiner Zeit auf Grund eigener Erlebnisse und Erkenntnisse, 191.

93 IMT, 29, 309. 
che una linea troppo aggressiva poteva causare all'economia di guerra. Servivano quindi provvedimenti di utilità pratica, che non si scontrassero apertamente con le disposizioni del Fübrer ${ }^{94}$ Secondo Frank il GG sarebbe dovuto diventare l'esempio di verbesserte Technik des Staates ${ }^{95}$ (tecnica perfezionata dello stato), un progetto a lungo termine di formazione per i nuovi funzionari di governo del Reich e dei territori occupati. Furono però le SS a occuparsene, educando soldati e ufficiali all'esecuzione di ordini brutali senza alcuno scrupolo. ${ }^{96}$ Solo dopo la bocciatura da parte di Hitler delle sue proposte, volte a sostituire una politica di pericolosa oppressione del popolo polacco con provvedimenti di utilità pratica, Frank tenne una condotta prudente ma continuamente interferita dalla linea "terroristica" tenuta da Krüger e, per reazione, da Frank stesso."

A causa dei continui conflitti di competenza e della mancanza di una politica di amministrazione chiara, nell'estate 1942 la posizione di Frank appariva pregiudicata. Accusato di appropriazione indebita, fu sottoposto ad un umiliante interrogatorio da parte delle SS di Krüger, il quale ottenne la nomina a segretario di stato e plenipotenziario per il "trapianto" di famiglie tedesche nella Polonia. ${ }^{98}$ La rivalità toccò il culmine nell'estate 1942, quando Frank tenne quattro discorsi in altrettante università tedesche (Berlino, Vienna, Monaco, Heidelberg) dal titolo Das Recht als Grundlage der Volksgemeinschaft (Il diritto come fondamento della comunità popolare). ${ }^{99}$ Il 20 luglio a Monaco sentenziò che «sarebbe [stato] inconcepibile un Reich senza diritto o in contrasto con il diritto ${ }^{100}$ e sostenne la necessità di avere come tutori della legge dei giudici indipendenti. ${ }^{101}$ Frank mostrò inoltre malcontento per l'insufficienza delle istituzioni esistenti e per il basso livello della loro organiz-

94 Cfr. J. Fest, Il volto del Terzo Reich, 341.

95 H. Frank, Technik des Staates. Schriftenreibe des Institutes für die Technik. des Staates an der Technischen Hochschule München. Heft I, a cura di E. Rudolf, in Zeitschrift für die gesamte Staatswissenschaft - Journal of Insitutional and Theoretical Economics 4, 1021942, 740-2.

96 J. Fest, Il volto del Terzo Reich, 339.

97 Ibid.

98 Ibid., 341.

99 Ibid.

100 Ibid., 333.

101 E. Davidson, Gli imputati di Norimberga. Hermann Wilhelm Göring, Rudolph Hess, Martin Bormann, Albert Speer... La vera storia di ciascuno dei ventidue fedelissimi di Hitler processati per crimini contro l'umanità dal Tribunale alleato, 464. 
zazione: l'aver copiato in parte la struttura burocratica del Reich «priva di anima» e «razionalmente rarefatta» rendeva l'attività amministrativa nel GG difficoltosa. ${ }^{102}$ Per tutta risposta, Hitler gli tolse la possibilità di fare discorsi in pubblico e lo destituì da tutte le cariche del partito. ${ }^{103}$ Non lo depose però dalla carica di Governatore e non accettò le sue dimissioni in nessuna delle quattordici volte in cui le presentò dal luglio 1942 al settembre 1944. A essere liquidato fu inaspettatamente Krüger, in seguito all'ondata di ribellione suscitata dai metodi brutali con cui veniva organizzata dalle SS la deportazione dei lavoratori coatti in Germania. Hitler riconobbe indirettamente con questa revoca la latenza dell'apparato statale nel GG. ${ }^{104}$

Con il successore di Krüger, lo SS-Obergruppenfübrer Wilhelm Koppe, Frank stabilì un rapporto di fredda collaborazione; gli attriti rimasero per questioni non personali ma strutturali del sistema di governo del GG.

La prospettiva della sconfitta in guerra e la sua paura di veder fallire completamente la sua esperienza di Governatore accentuarono in Frank le sue insicurezze, evidenti nella sua tendenza a prendere provvedimenti che ne smentivano altri precedenti. Per esempio, a partire dal 1944 propose una politica di «umanità» e di «europeizzazione», senza però riuscire con questi tardivi rimedi né a convincere Hitler né a ripulirsi la reputazione presso la popolazione polacca. Il 18 agosto 1944 informava Berlino della «completa dissoluzione dell'autorità» della sua amministrazione e proponeva lo scioglimento del GG. ${ }^{105}$ In uno degli ultimi discorsi ai propri collaboratori al castello di Cracovia pregava di «non soffocare fino in fondo gli elementi vitali insiti nel sangue di un popolo». ${ }^{106}$

A Norimberga Frank descrisse gli anni da Governatore come i più terribili della sua vita, spiegando come fosse «un uomo isolato, privo di potere, senza alcun influsso sugli avvenimenti». ${ }^{107} \mathrm{La}$ insoddisfazione del

102 J. Fest, Il volto del Terzo Reich, 339.

103 E. Davidson, Gli imputati di Norimberga. Hermann Wilhelm Göring, Rudolph Hess, Martin Bormann, Albert Speer... La vera storia di ciascuno dei ventidue fedelissimi di Hitler processati per crimini contro l'umanità dal Tribunale alleato, 464.

$104 \mathrm{~J}$. Fest, Il volto del Terzo Reich, 342.

105 Ibid., 342-3.

106 IMT 29, 717.

107 H. Frank, Im Angesicht des Galgens. Deutung Hitlers und seiner Zeit auf Grund eigener Erlebnisse und Erkenntnisse, 394. 
Governatore riguardò l'incapacità di avere effettivamente i pieni poteri amministrativi che la natura della sua carica avrebbe dovuto attribuirgli; il disagio del giurista era più profondo, non riusciva a conciliare la vocazione di un uomo del diritto in un paese privo di organizzazione e di un ordinamento logicamente strutturato, dove il capo supremo (Hitler) pensava che «nessuno era più affine ai giuristi quanto i criminali». ${ }^{108}$

Scrisse in carcere: «Era mio fermo intendimento obbedire alle idee di Hitler, rifiutando però i suoi metodi. Ma io avrei dovuto dire no alle sue idee: avrei così evitato di trovarmi immerso in questa contraddizione». ${ }^{109}$

Frank fu una figura caratterizzata dal dubbio e dall'incertezza e, diviso fra l'influenza hitleriana e la sua posizione di "uomo di legge", non riuscì a mantenere il contatto con la realtà e a governare una colonia troppo dipendente dalla madrepatria. L'attività di buon amministratore e di inventore di un nuovo «diritto comunitario tedesco» non furono mai realizzate e il suo Governatorato rispecchiò e, per certi versi, ingigantì i difetti dei "doppi livelli istituzionali" dello stato nazionalsocialista.

\section{OrganizZAZIONE ISTITUZIONALE E SOCIALE}

Il GG si divideva in dodici dipartimenti (arrivarono anche a quattordici), gli Hauptabteilungen, e una segreteria di stato con diversi uffici (operativi però dal marzo 1941). C'erano undici plenipotenziari distaccati dal Reich in rappresentanza dei vari ministeri interessati. Le altre autorità politiche che esercitavano effettivamente il potere erano i funzionari locali, che in base ai diversi gradi di territorialità governavano la contea (Kreis), la città (Stadt) o il distretto (Distrikt). L'utilizzo nel GG della stessa struttura burocratica del Reich agevolò l'attività dei ministri tedeschi che potevano avere contatti diretti con le singole amministrazioni locali, senza dover rendere conto al Governatore. Le uniche due materie sulle quali Frank doveva essere sempre informato erano le comunicazioni e le poste, ma anche in questo caso agiva spesso di concerto con la Gestapo. ${ }^{110}$

108 H. Picker, Hitlers Tischgespräche im Führerhauptquartier 1941-1942, Ullstein, Bibliothek der Zeitgeschichte, 1989, 260.

109 H. Frank, Im Angesicht des Galgens. Deutung Hitlers und seiner Zeit auf Grund eigener Erlebnisse und Erkenntnisse, 186.

110 J.T. Gross, Polish society under German occupation. The Generalgovernment 1939-1944, Princeton University Press, 1979, 51-2. 
Il 12 ottobre fu emanato il primo atto amministrativo per i territori occupati: nell'atto costitutivo del GG Hitler escludeva i territori che nel gennaio 1940 sarebbero poi stati annessi al Reich e nominava Frank Governatore. Furono abolite le istituzioni amministrative del dissolto stato polacco, il diritto polacco rimase in vigore dove risultava più utile ai tedeschi e dove non contrastava con i principi del Reich. ${ }^{11}$ Il GG ricevette regole diverse rispetto ai territori annessi alla Germania sul fronte interno: ordine pubblico, barriere economiche, trattamenti sanitari, rigorosi controlli doganali. Sebbene il GG non avesse un proprio esercito o un proprio ministro degli esteri, si dotò di una sorta di ambasciatore a Berlino, anche per dare un più alto valore istituzionale alla carica di Frank. ${ }^{112}$ Dopo il 1941 tutti i funzionari locali, a partire dalle contee, vennero subordinati ad un Hauptmann o a un Governeur locali, causando una perdita di potere dei politici locali e un peggioramento della gestione anche se gli interessi dei singoli prevaricavano spesso le direttive nazionali. Col dilagare della corruzione, i responsabili tedeschi favorivano gli interessi anche dei polacchi che potevano permettersi di pagare; come nel caso della raccolta delle tasse nel 1940 a Varsavia, che avrebbe dovuto produrre un gettito da versare all'amministrazione del GG e che invece rimase nelle casse cittadine per volontà del dipartimento delle finanze della città. ${ }^{113}$

La disorganizzazione della burocrazia fu aggravata dal fatto che gli uffici della segreteria di stato raddoppiavano il lavoro e i livelli burocratici anziché snellire i procedimenti. Gli uffici economici tentarono deliberatamente di eliminare le strutture produttive polacche rimaste ancora attive dopo il decreto di Göring, mentre gli uffici per l'industria lavoravano per il loro mantenimento, a consolidamento dell'economia del territorio. Anche sul piano legislativo c'era un ufficio alla segreteria di stato e un dipartimento di giustizia con gli stessi compiti: l'amministrazione ne risultava così bloccata e alcune materie importanti (come l'agricoltura) ricevevano solo l'indirizzo dell'autorità centrale del GG. ${ }^{114}$

Durante un incontro del 18 giugno 1942 le autorità ministeriali e

111 Cfr. NCA 4 (ND, 2233-A-PS - 2233-CC-PS), in http://avalon.law.yale. edu/imt/2233-ps.asp (05-05-2015).

112 J.T. Gross, Polish society under German occupation. The Generalgovernment 1939-1944, 46

113 Ibid., 52, nota 7.

114 Ibid., 52-3. 
dipartimentali decisero una semplificazione delle procedure burocratiche, con lo scopo di attribuire incisività alle decisioni a livello locale con una sorta di decentralizzazione. Frank, già politicamente in difficoltà, si oppose temendo di perdere buona parte del proprio potere. La mancata semplificazione amministrativa comportò la paralisi decisionale all'interno del GG, che divenne insostenibile dopo la "scomunica" hitleriana a Frank dell'estate 19421.115

La dominazione nazista dell'Europa si identificava nel Nuovo Ordine, sistema di gestione della cosa pubblica caratterizzato da un metodo disorganizzato, sul piano giuridico e sociale, di amministrare il territorio. Il primo obiettivo era la cancellazione delle istituzioni politiche democratiche e la distruzione del pluralismo nella vita sociale: partiti politici, organizzazioni sindacali e libere associazioni culturali scomparvero in Germania senza forti resistenze a partire dal 1933 in ottemperanza alla Gleichschaltung (allineamento), punto di riferimento del programma nazionalsocialista. ${ }^{116}$ Anche nel GG le organizzazioni polacche furono soppresse e la maggior parte della popolazione fu assoggettato alle pratiche nazionalsocialiste; ma l'esigenza di sopravvivere all'esperienza autoritaria finì col portare a due scelte differenti. La prima era mantenere lo spirito di resistenza all'invasore e organizzarsi in movimenti politici e culturali clandestini. La seconda fu assecondare l'occupante tedesco nel perseguimento di alcuni obiettivi, come il mantenimento dell'ordine pubblico, il reclutamento del lavoro coatto da spedire in Germania e il controllo sulla produzione agricola, in gran parte destinata al mercato tedesco. La seconda scelta si verificò specialmente nel primo anno e mezzo di occupazione, quando la situazione era grave ma ancora sostenibile per la popolazione e quando il debole governo di Frank riusciva ancora a prendere decisioni senza le interferenze delle SS di Krüger. ${ }^{117}$

La cooperazione posta in essere da molte organizzazioni e da liberi cittadini indebolì la società civile portando alla sostituzione della struttura socio-economica e culturale polacca con una forma disorganizzata di amministrazione germanica. Sebbene Frank teorizzasse le basi giuridiche

115 J.T. Gross, Polish society under German occupation. The Generalgovernment 1939-1944, 53.

116 Ibid., 29.

117 E. Davidson, Gli imputati di Norimberga. Hermann Wilhelm Göring, Rudolph Hess, Martin Bormann, Albert Speer... La vera storia di ciascuno dei ventidue fedelissimi di Hitler processati per crimini contro l'umanità dal Tribunale alleato, 446-66. 
nazionalsocialiste e l'applicazione nel GG dei crismi del «diritto comunitario tedesco», l'immobilismo politico della sua gestione e i conflitti di competenza con le autorità di polizia favorirono l'instabilità sociale, che necessitava invece di una struttura statale equilibrata, unica soluzione per dare una legittimazione giuridica all'esistenza di questa entità statuale. ${ }^{118}$

Le sovrapposizioni tra i poteri (che Hitler non aveva alcun interesse a risolvere visto che traeva forza dalle liti fra i suoi subordinati) era tipica del sistema amministrativo e di governo nazionalsocialista; anche nel GG questa anomalia riguardava sia i rapporti tra poteri statali, sia i rapporti tra organismi statali e di partito, sia tra potere politico e polizia. Per esempio, le circolari di Himmler e i relativi "decreti attuativi" di Heydrich e Krüger sul lavoro coatto spesso entravano in contrasto con i decreti del Governatore; pertanto l'unico modo per risolvere la diatriba era ricorrere al giudizio finale del Fübrer. Questo sistema rafforzava la figura e l'autorità del dittatore ma produceva conseguenze gravi e a volte insanabili sulla gestione degli "affari correnti” del GG perché rendeva difficile, anche dopo la decisione di Hitler, stabilire quale compito spettasse a chi e in che misura. ${ }^{119} \mathrm{Il}$ perseguimento da parte di Frank delle finalità indicate dal Nuovo Ordine, nel rispetto e nell'attuazione dei parametri del diritto nazionalsocialista risultava impossibile poiché mancava la possibilità di una effettiva centralizzazione del potere e ottimizzazione delle risorse. ${ }^{120}$ Le uniche circostanze in cui la confusione interna al GG si tramutava in una inaspettata efficienza ed efficacia negli atti amministrativi erano quelle in cui gli ordini arrivavano direttamente da Hitler e l'unica cosa da fare era rispettare la "volontà del Führer". ${ }^{121}$ Si può quindi affermare che «il potere nazista e la sua istituzionalizzazione [nel GG] permisero una effettiva ed efficace mobilitazione per obiettivi a corto raggio e con breve preavviso». ${ }^{122}$

Lo scarso livello del corpo istituzionale del GG favorì una certa

118 J.T. Gross, Polish society under German occupation. The Generalgovernment 1939-1944, 30, e in I. Kershaw, Hitler e l'enigma del consenso, 100-11.

119 J.T. Gross, Polish society under German occupation. The Generalgovernment 1939-1944, 31.

120 Ibid.

121 Cfr. I. Kershaw, Hitler e l'enigma del consenso, 100-11.

122 J.T. Gross, Polish society under German occupation. The Generalgovernment 1939-1944, 31 
elasticità nel sistema nazionalsocialista e consentì l'attuazione della logica di perseguire gli obiettivi di governo con l'incisività non riscontrabile in un sistema democratico, ma nemmeno nello stesso Governatorato quando l'iniziativa "legislativa" partiva dal suo interno. ${ }^{123}$ Ogni progetto politico a lungo termine, fatta eccezione per le operazioni di sterminio, era invece irrealizzabile.

Secondo Czeslaw Madajczyk ${ }^{124}$ la qualità del personale amministrativo impiegato in Polonia dai nazisti era molto bassa; il personale più preparato veniva destinato ai territori occupati dell'Europa occidentale. ${ }^{125}$ Hitler insisteva affinché «le personalità forti», cioè i nazisti più convinti, venissero mandate a est, assieme ai neo-laureati dell'Ordensburg, ${ }^{126}$ giovani militanti che entravano in questa scuola di indottrinamento fondata dal direttore del fronte del lavoro Robert Ley. I quadri del partito formatisi in questo istituto avrebbero dovuto rappresentare il futuro politico del NSDAP e del Reich; in realtà erano, a parere di taluni, inadeguatamente preparati. Assieme al personale selezionato dal partito si trovavano nelle amministrazioni avvocati inattivi, sindaci e funzionari senza poltrona, non all'altezza di un posto di buon livello e impiegati nell'Ostgebiet ${ }^{127}$ meno prestigioso per la carriera dei territori occidentali occupati. A partire dalla fine del 1940 le autorità del governo centrale, delle città e delle contee furono costrette all'affannosa ricerca di personale. Ludwik Landau riporta un documento dell'amministrazione comunale di Lodz in cui si invitava qualsiasi candidato ad iscriversi all'elenco per ogni posizione si volesse ricoprire. ${ }^{128}$ Secondo Alfred Rosenberg, ministro per l'Ostland dal luglio 1941, «il governo tedesco nella Polonia occupata fu dal principio inefficace, sia sul piano politico sia su quello amministrativo», i territori orientali

123 Ibid., 32.

124 Storico polacco (1921-2008).

125 Cfr. C. Madajczyk, Polityka III Rzeszy w okupowanej Polsce, okupacja Polski 1939-1945, Warsaw, PWN, 1, 1970, 497, in J. T. Gross, Polish society under German occupation. The Generalgovernment 1939-1944, 54.

126 D. Orlow, The History of the Nazi Party, Pittsburgh, University of Pittsburgh Press, 1973, 188-92.

127 NCA 4 (NS, 2220-PS), in http://avalon.law.yale.edu/imt/2220-ps.asp (0505-2015).

128 J.T. Gross, Polish society under German occupation. The Generalgovernment 1939-1944, 55. 
erano un covo di corrotti e di incapaci e il GG era «sinonimo di regime coloniale impazzito». ${ }^{129}$

La sezione "polacca" del NSDAP, istituita da Frank nel maggio 1940, sarebbe dovuta essere una via di mezzo tra il modello tedesco di partito e quelli degli altri territori occupati (Heimatsgau und Auslandsgau) e prese il nome di Arbeitsbereich GG der NSDAP (zona di lavoro nazionalsocialista). ${ }^{130}$ Frank, i capi-distretto e i capi-quartiere (in polacco szefpowiat) sommarono anche le cariche di guida delle rispettive sezioni territoriali del partito (Personalunion). ${ }^{131}$ Nonostante l'unione delle cariche la dimensione partitica prese il sopravvento sull'amministrazione dello stato e le questioni dei singoli nel partito venivano risolte utilizzando le istituzioni statali e locali. A causa di questa gestione il partito non era in grado di svolgere una funzione di garanzia e controllo sull'attività di governo, ma ne divenne anzi uno strumento di disturbo. ${ }^{132}$

In Polonia si ebbe una "espansione aggressiva" in quattro gradi: geografica, economica, assimilativa e ideologica. ${ }^{133}$ L'idea che le nazioni non fossero semplicemente unità politiche e territoriali distinte ma che potessero essere distinte tra "migliori" e "peggiori" denotava una implicita valutazione negativa delle mescolanze razziali, in contrasto con i provvedimenti presi sui livelli di appartenenza o classificazioni. Il nazionalsocialismo mirava inizialmente solo al trionfo della razza ariana nei confronti degli Untermenschen; le evoluzioni che portarono alle Mischehen furono il frutto di una interpretazione della dottrina. ${ }^{134}$

La dominazione tedesca nel GG fu influenzata dai principi ideologici del partito e dal "realismo politico nazionalsocialista" che gli uomini di potere, Frank su tutti, cercarono di applicare. Il governo di Frank fu chiamato a gestire i rapporti tra gli occupanti e gli abitanti delle aree invase e la "destinazione d'uso" di questi ultimi.

Vi erano infatti due tipi diversi di procedure per attuare la rivolu-

129 D. Orlow, The History of the Nazi Party, 297.

130 J.T. Gross, Polish society under German occupation. The Generalgovernment 1939-1944, 57.

131 Ibid.

132 D. Orlow, The History of the Nazi Party, 293.

133 F. Znaniecki, Modern nationalities: a sociological study, Urbana, University of Illinois Press, 1952, 114-6.

134 Cfr. J.T. Gross, Polish society under German occupation. The Generalgovernment 1939-1944, 35. 
zione nazionalsocialista nei territori occupati: la politica di annientamento immediato dei popoli inferiori (Vernichtungspolitik), secondo il principio della tabula rasa, oppure la ridefinizione delle teorie razziali secondo un'analisi pragmatica della popolazione che si traducesse nel sistema delle classificazioni. In questo senso, la politica razziale europea dei nazisti si orientò, per volontà di Himmler, verso l'opportunità di un'assimilazione degli elementi caratterizzati da una «purezza incompleta». ${ }^{13}$ Questa soluzione si trasformò in una fonte di arricchimento per il Reich, perché i cittadini ammessi accrebbero la forza lavoro e il numero degli abili alla leva; contemporaneamente, per gli esclusi da questo elenco, soprattutto gli ebrei, venne applicata subito la soluzione dei ghetti..$^{136}$

Il 20 ottobre 1939 si svolse a Berlino una conferenza tra Hitler e l'alto comando della Webrmacht in cui venne regolata la politica tedesca di occupazione articolandola in tre punti. Per prima cosa, la Germania decise di non incorporare il GG, che sarebbe stato considerato formalmente come uno stato indipendente; alle autorità tedesche conveniva avere uno stato-colonia separato da Berlino, nel quale poter fare indisturbati «ciò che dovevano»: non è infatti un caso che nel GG non fu introdotta la legislazione penale tedesca. ${ }^{137}$ In secondo luogo, furono perseguiti con puntiglio due obiettivi imprescindibili per il funzionamento del nuovo stato: impedire all'inteligencja polacca di esercitare un ruolo guida per il futuro e conservare in buone condizioni - e nel caso ampliare - le linee di comunicazione affinché il GG potesse essere utilizzato come agile via di passaggio per le conquiste future in oriente. Infine, sul medio-lungo periodo, il GG avrebbe dovuto rappresentare il punto nevralgico delle operazioni di "epurazione" di ebrei e slavi.

Il governo Frank attuò provvedimenti di natura preventiva: evitare la rinascita di un orgoglio nazionale polacco e di un'economia indipendente dagli ordini delle autorità tedesche, favorendo la Polnische Wirtschaft. ${ }^{138} \mathrm{I}$ polacchi dovevano servire solo come manodopera coatta, senza alcun potere di iniziativa, se non quello di offrirsi volontari per gli "spostamenti" in Germania.

135 Gruppi (o livelli) 2, 3 e 4 del sistema di classificazione razziale, vedi nota 26.

136 J.T. Gross, Polish society under German occupation. The Generalgovernment 1939-1944, 40-1.

137 Ibid., 46.

138 Ibid., 47. Polnische Wirtschaft è un'espressione ironica e fortemente dispregiativa con cui i tedeschi definivano il grado "inferiore" dell'economia dei polacchi del GG. 
La prima direttiva in ambito industriale fu emanata da Göring il 13 ottobre 1939: come plenipotenziario per il piano quadriennale ordinò che venissero trasferite materie prime, macchinari, materiali di scarto nelle industrie tedesche per essere impiegate nell'economia di guerra. Le aziende che non servivano alla produzione di beni essenziali al mantenimento della popolazione dovevano essere tassativamente trasferite in Germania, ${ }^{139}$ nonostante molte delle delocalizzazioni comportassero un irragionevole lungo periodo e costi elevati. $^{140}$

Quando il 26 ottobre 1939 assunse pieni poteri - nei limiti della natura della sua carica - Frank prospettò ai polacchi sotto la sua amministrazione un roseo futuro, con la promessa che avrebbero potuto coltivare la loro Polentums ${ }^{141}$ ("polonità") in una nuova atmosfera e sotto la protezione del Grande Reich tedesco. Non era conosciuto dalla popolazione locale l'obiettivo della Wiedereindeutschung, ossia la completa germanizzazione dei territori polacchi annessi al Reich mediante il sistema delle classificazioni. Questo fu l'ultimo passaggio di "scrematura" dei cittadini polacchi prima di dare inizio alle operazioni di «morte attraverso il lavoro» o di sterminio: ${ }^{142}$ le procedure, aventi secondo Himmler una qualche base scientifica, furono condotte con criteri personalistici e di convenienza da parte delle autorità tedesche. ${ }^{143} \mathrm{Ne}$ è un esempio il $3 \%$ della popolazione polacca ritenuta adatta alla germanizzazione, oppure il fatto che quasi tutti i cechi residenti nel GG vennero iscritti alla DVL ad opera dei reparti medici delle SS. I diversi responsabili di zona delle SS applicavano quindi le regole di Inklusionen ed Exklusionen in maniera non oggettiva, giustificando il rilascio di permessi "generosi" con l'urgenza e la necessità di un esito favorevole del processo di germanizzazione («ein rechtlicher Zustand der dem gerade bei ibnen dringend notwendigen Eindeutschungsbestreben wenig förderlich ist»)..$^{144}$

139 NCA, 4 (NS, 1665-PS), in http://avalon.law.yale.edu/imt/1665-ps.asp (0605-2015).

140 J.T. Gross, Polish society under German occupation. The Generalgovernment 1939-1944, 48.

141 Cfr. B. Kundrus, Regime der Differenz. Volkstumspolitische Inklusionen und Exclusionen im Warthegau und im Generalgouvernement 1939-1944, 110-7.

142 Ibid.

143 Ibid., 114-5.

144 Ibid. 
Nel marzo 1941 fu pubblicato sulla Reichsgesetzblatt (Gazzetta Ufficiale del Reich) un decreto di Himmler in cui si attribuiva la cittadinanza tedesca, tolta a chi non ne era più degno, ai cittadini di varie razze che rispettavano i parametri e le direttive stabiliti dallo stesso Himmler, in qualità di Commissario del Reich per il rafforzamento della germanicità. ${ }^{145}$

\section{Aspetto ECONOMico E DEL LAVORO}

Anche sul piano economico non c'era una visione organica del sistema statale da parte delle autorità politiche del GG. Gli unici dati certi riguardavano da un lato l'interesse dei grandi capitalisti tedeschi a mantenere in Germania o spostare in Polonia l'industria pesante tedesca per avere a disposizione la bassa manovalanza polacca e dall'altro la funzione di granaio del GG per le esportazioni di prodotti agricoli nel Reich ${ }^{146}$ veniva quindi attuato uno sfruttamento «nel senso meno sofisticato del termine». ${ }^{1.7}$

La gestione degli amministratori del GG causò il ripetersi di operazioni non redditizie all'interno del territorio: la maggior parte delle attività industriali e della produzione energetica dell'ex-stato polacco era concentrata nei territori occidentali e settentrionali, annessi al Grande Reich tedesco. Quasi tutti i macchinari e gli impianti presenti nel GG furono smontati e portati in Germania nei primi mesi di occupazione. Inoltre, pur essendo stata la Polonia un paese esportatore di prodotti agricoli prima della guerra, nei circa cinque anni di Governatorato non venne mai raggiunta l'autosufficienza alimentare. ${ }^{148}$

Le prime direttive generali per lo sfruttamento del GG furono dettate il 13 ottobre 1939 da Göring, come plenipotenziario del Reich per l'attuazione del piano quadriennale; successivamente all'integrazione tramite decreto del novembre dello stesso anno, si stabilì la possibilità dei tedeschi di appropriarsi di tutte le strutture, gli impianti e i beni (anche artistici) di proprietà del soppresso stato polacco. ${ }^{149}$

145 NCA 5, 587-90 (NS, 2917-PS).

146 A. Milward, The German Economy at War, London, University of London The Athlone Press, 1970, 30.

147 Ibid., 31.

148 J.T. Gross, Polish society under German occupation. The Generalgovernment 1939-1944, 92.

149 Ibid., 93. 
Due decreti distinti si occupavano di espropriazione (Beschlagnabmt) e confisca (Einziebung): non c'erano grandi differenze sul piano pratico, che si basava sulla privazione dei polacchi del diritto di proprietà. Il 15 novembre 1939 un decreto del Governatore ordinò il passaggio di proprietà al GG di tutti i beni sottratti ai polacchi. ${ }^{150}$ La legittimazione giuridica fu quella del "pubblico interesse", ribadita nel decreto attuativo del gennaio 1940. I capi-distretto e le figure istituzionali indicate specificamente da Frank avevano il potere di esproprio e confisca.

Questa grande operazione avrebbe dovuto arricchire il GG attraverso l'acquisizione di beni di varia natura ma, a causa delle modalità con cui venivano effettuate le confische e i sequestri o congelati i conti bancari, l'equilibrio distributivo della ricchezza venne meno assieme alle possibilità di lavoro per molte categorie. Inoltre tutte le istituzioni che avevano prestato denaro allo stato polacco o alle imprese persero il diritto alla riscossione del credito e fallirono. Per i polacchi invece la responsabilità in solido per le obbligazioni era stata aggravata. ${ }^{151}$

Tra il 1939 e il 1942 furono prese misure fiscali importanti: il Reichsmark fu sopravvalutato nello scambio con lo ztoty del $33 \%$, modificando il tasso di cambio per fermare il flusso di trasferimenti di capitali all'estero; i conti bancari individuali vennero congelati e fu concessa la possibilità di prelevare in pochi casi e per importi bassi. L'amministrazione statale perse circa 800 milioni di złoty. ${ }^{152}$

La popolazione polacca impoverì in modo preoccupante e in poco tempo, tanto da allarmare anche le autorità tedesche stesse; con l'inizio della campagna di Russia diminuirono ulteriormente i fondi da destinare alla sopravvivenza della popolazione polacca, la quale si vide invece aumentare l'imposizione fiscale: rispetto all'autunno 1939, nel 1942 tasse e accise di vario tipo che gravavano solo sui polacchi erano raddoppiate e aumentò anche l'inflazione. ${ }^{153}$ Il PIL diminuì del 40\% dal 1938 al 1941; l'approvvigionamento di generi

150 NCA 5 (NS, 1773-PS), in http://avalon.law.yale.edu/imt/1773-ps.asp (0605-2015).

151 J.T. Gross, Polish society under German occupation. The Generalgovernment 1939-1944, 94.

152 A. Milward, The German Economy at War, 54.

153 Ibid. 
alimentari dall'inizio del 1941 calò gravemente, al punto di essere insufficiente a coprire i fabbisogni della popolazione polacca. ${ }^{154}$

In una conferenza a Cracovia il 24 agosto 1942 Frank espresse soddisfazione per la diminuzione dell'apporto calorico medio procapite per la popolazione: ogni risorsa impiegata nell'approvvigionamento di generi alimentari sarebbe stata - a suo dire - tolta ad altre attività di interesse tedesco, quindi ogni privazione alla popolazione andava salutata con favore. ${ }^{155}$

Tra le voci che formavano il PIL del GG l'agricoltura era la più importante. La terra era infatti una delle cinque grandi direttrici della dottrina nazionalsocialista, assieme a razza, lavoro, Reich e onore. Il principio di tutela della terra che «sfama il popolo» e dei contadini che «lavorano la terra» era già disciplinato da una legge del Reich del 29 settembre 1933 che istituiva i poderi ereditari per «garantire per sempre l'esistenza delle famiglie contadine». ${ }^{156}$ Per Frank però non era necessaria anche la conservazione delle famiglie contadine polacche, perché se l'obiettivo era lo sfruttamento delle risorse per il "bene comune" del popolo tedesco, i lavoratori della terra sarebbero serviti solo fino alla fine della guerra; poi la terra sarebbe andata ai vincitori. ${ }^{157}$

Durante il primo anno di occupazione le condizioni di vita nelle campagne migliorarono. I debiti verso i finanziatori ebrei furono cancellati, le imposizioni fiscali nelle zone agricole e rurali incidevano in misura minore rispetto alle città. I prezzi dei prodotti agricoli aumentavano più degli altri prodotti e i salari dei contadini erano più alti di quelli degli operai e quindi il tenore di vita nelle campagne era proporzionalmente elevato. ${ }^{158}$ Nella vita quotidiana il cambiamento generò anche effetti negativi, come la piaga dell'alcolismo, che non era mai stato così grave come durante la guerra. ${ }^{159}$

La popolazione polacca doveva convivere con il problema della

154 J.T. Gross, Polish society under German occupation. The Generalgovernment 1939-1944, tabella IV.4, 99.

155 NCA 4, 893-902 (NS-2233-E-PS).

156 H. Frank, Fondamento giuridico dello Stato nazionalsocialista, 36.

157 Ibid.

158 J.T. Gross, Polish society under German occupation. The Generalgovernment 1939-1944, 104.

159 Ibid., 105. 
distribuzione dei viveri e con le differenze con i tedeschi. I polacchi, da 10 a 12 milioni di persone fino al 1941, ricevevano meno della metà del totale delle forniture al netto delle spedizioni in Germania e ricorrevano spesso al mercato nero. Attraverso la corruzione di alcuni funzionari nazisti compiacenti, i produttori accantonavano illecitamente le riserve che alimentavano il mercato illegale.

L'economia sommersa accrebbe il proprio volume a causa della povertà e della malnutrizione della popolazione. Il sistema era rischioso e dispendioso sia per i molti funzionari da corrompere, sia per il tempo usato nell'organizzare i trasporti delle merci indebitamente accantonate a riserva. Tutte le attività commerciali legali ricevevano un'autorizzazione supplementare per sopportare il doppio binario. ${ }^{160}$

I datori di lavoro tenevano due tabelle salariali diverse e due tipologie di contabilità. I salari, fermi ufficialmente all'ante guerra, erano potenziati fino ad arrivare a 35 ztoty all'ora (circa il quadruplo del normale) per alcune mansioni. I salari così alti nel mercato nero provocarono anche il $30 \%$ di assenteismo nei posti di lavoro "regolari". ${ }^{161}$

Le autorità tedesche non riuscirono a mantenere il lavoro nei binari determinati dalla legge del Reich, che si ispirava alla «legge sul lavoro nazionale» del 20 gennaio 1934. La natura razziale "inferiore" dei polacchi determinò la schiavizzazione dei lavoratori, gli operai più dei contadini. ${ }^{162}$ Furono soppressi i diritti dei lavoratori e l'Arbeitsamt (dipartimento del lavoro) gestiva la materia a sua totale discrezione. La costruzione di alcune opere pubbliche di utilità per i tedeschi (opera indicata come Polnischer Baudienst im Generalgouvernement, o semplicemente Baudienst) fu anzi una dimostrazione di sfruttamento del lavoratore coatto, figura introdotta nella legislazione nel dicembre $1940,{ }^{163}$ poi rivista con il decreto del Führer sulla nomina del «plenipotenziario generale sull'utilizzazione del lavoro» del 21 marzo 1942. ${ }^{164}$ Inizialmente si trattava di giovani dai 18 ai 25 anni "reclutati"
160 Ibid., 109.
161 Ibid., 110.
162 Ibid.
163 H. Frank, Fondamento giuridico dello Stato nazionalsocialista, 39.
164 NCA 4 (ND, 1666-PS), in http://avalon.law.yale.edu/imt/1666-ps.asp (1005-2015). 
per tre mesi (allungati a sette). I prescelti erano sottoposti ad una sorta di addestramento militare per imparare a svolgere il ruolo di Vorarbeiter (capo squadra o supervisore), che godeva di un trattamento economico di livello più alto. Lo scopo del Baudienst era quello di insegnare ai polacchi «lavoro e disciplina». ${ }^{165}$

\section{CONCLUSIONI}

Sul piano giuridico Frank puntava a dare al suo stato una disciplina giuridica onnicomprensiva basata sui principi del "suo" diritto; diede quindi il via all'attuazione di un processo di legittimazione sia medico-scientifica sia giuridica in senso stretto dell'ideologia nazista al fine di istituzionalizzarla in uno Stato (almeno formalmente) autonomo rispetto alla Germania. La suddivisione nei quattro gruppi stabiliti dal decreto di Himmler del 12 settembre 1940 e il regolamento sulle DVL nell'Ostgebiet del 4 marzo 1941 sanciscono l'esportazione della dottrina nazista con lo scopo di separare gli ariani "puri" dai Volksgenossen im Lemprozess, ossia gli appartenenti al cantiere razziale da cui il Reich avrebbe attinto per "rinfoltire i ranghi". La direzione del RKFDV serviva per la selezione di giovani "tedeschi" da spedire al fronte e donne geneticamente associabili alla razza ariana per la procreazione e l'ampliamento della Großdeutsche Volksgemeinschaft.

Il mutare del peso della figura del Governatore all'interno del sistema istituzionale seguì la parabola discendente della sua già precaria esperienza politica: al centro dei processi decisionali per il primo anno di vita del nuovo stato, fu chiaro al primo giureconsulto del Reich che i vincitori dei conflitti di competenze tra le autorità operanti nel GG furono le SS e che Frank si trovò col passare dei mesi solo formalmente al comando. Riguardo l'amministrazione interna dello stato, Frank si dimostrò impotente nel contrastare il ruolo delle SS nella gestione del lavoro coatto e, in generale, del "materiale umano" contenuto nel GG. Ciononostante la sua figura ebbe un ruolo essenziale nell'analisi del sistema giuridico e istituzionale nazionalsocialista

165 J.T. Gross, Polish society under German occupation. The Generalgovernment 1939-1944, 108-110. 
e dei rapporti tra poteri dello stato. Per quanto riguarda l'amministrazione interna del GG, Frank si dimostrò impotente nel contrastare il ruolo delle SS nella gestione del lavoro coatto.

Gli atti giuridici del Governatore furono di natura violenta e repressiva, in contraddizione con la volontà espressa da giurista di porsi a capo di un progetto per la creazione di un «diritto comunitario tedesco».

I difetti istituzionali del GG furono ingigantiti dalle inadeguatezze di Frank come amministratore e burocrate e lo stato si "ammalò" degli stessi "doppi livelli istituzionali" da cui era affetta la Germania.

Infine, la scarsa considerazione di cui godevano i giuristi presso Hitler ridimensiono il prestigio e il raggio di azione di Frank, ridotto al ruolo di fantoccio deriso dalle SS e privo dei poteri sognati, che fini col concentrarsi sulla violenza per affermare la sua fedeltà al Fübrer e la sua appartenenza alla nomenclatura di primo livello del partito. 